\title{
Intelligent and Hybrid Systems of Process Control: Theory, Methods, Applications \\ Suleimenov Batyrbek Aitbayevich
}

\author{
Doctor of Technical Science, Professor of the K.I. Satpayev Kazakh National Technical University, \\ Almaty city, Republic of Kazakhstan \\ Email: batr_sul@mail.ru
}

\section{Sugurova Laura Alkhaidarovna}

PhD student of the K.I. Satpayev Kazakh National Technical University, Almaty city, Republic of Kazakhstan

Email: sla-taraz@mail.ru

\section{Suleimenov Alibek Batyrbekovich}

PhD student of the K.I. Satpayev Kazakh National Technical University, Almaty city, Republic of Kazakhstan Email: alibek.suleymenov@mail.ru

Doi:10.5901/mjss.2015.v6n1s1p627

\section{Abstract}

This paper proposes a method of creating automated systems of optimal process control, based on using modern intelligent technologies. As shown by long-term experience, the traditional methods of mathematical modelling do not allow for the creation of effective system of optimal control due to their low adequacy. Instead of creation of mathematical models of process technologies, it is proposed to create intelligent models (algorithms) of control over process technologies, based on experience, knowledge and intuition of process control operators. Meanwhile, instead of generally accepted production rules, it is proposed to form a planning matrix of full factorial experiment (FFE). The planning matrixes of FFE are formed by execution of mental (thought) experiments, which significantly reduces the terms and simplifies the development of optimal control system. The proposed method has been tested on the basis of example on development of system on optimal control over the granulation (pelletizing) process - one of the most widely used in the metallurgical and chemical industries. Achieved algorithms showed sufficient high efficiency for practice.

Keywords: artificial intelligence, neural networks, fuzzy algorithms, neuro-fuzzy algorithms, optimal control, control algorithm, control system, pelletizing, granulation.

\section{Timeliness of the Topic}

Currently, development of system of optimal process technologies control in metallurgy, chemical industry, petrochemistry, etc., which could allow more efficient using of mineral resources, saving electrical and heating power, reducing environmental problems, increase economic returns from production. Rapid stage in the development of optimal control systems of different processes in the world, USSR and Kazakhstan took place in the 60-80 years of the last century. However, for example in Kazakhstan it is still not adopted any significant system of optimal control. This is due to extreme complexity of process technologies in nonferrous and ferrous metallurgy, chemistry and other sectors of economy of Kazakhstan. Attempts to create sufficiently adequate mathematical models of such complex processes, unfortunately, have not been successful, and trend for modeling gradually moved away. In recent years, publications in this direction have been significantly decreased.

However, rapid development of modern methods of development and creation of intelligent system brought to notable increase of publications on practical application of mentioned methods during creation of control system.

Meanwhile, a large share of such researches devoted to development of system of local control, aimed at solving of tasks on stabilization of some output variables by intelligent controllers. Taking this into account, there is a very urgent task on development of methods and means of creation of intelligent system on optimal process technologies control in conditions of market economy, which could significantly raise their economic efficiency. 
However, according to our opinion, it is more effective to use intelligent technologies in association with classic methods of process control. Meanwhile it is possible to combine advantages of traditional methods, techniques, and algorithms with mathematical tool of Al theory. Such systems we will call hybrid control system (HCS).

In different spheres of practical activity associated with solving of tasks of management and control, optimization and modelling, search and selection, recognition and classification, there is a demand in intelligent assistance to overcome difficulties in weakly formalized situations and with limited resources.

Methods and means of artificial intelligence are materialized and reach the consumer in form of intelligent technologies that are almost invariant with respect to one or another problem area. The present dissertation is devoted to consideration of issues on practical application of modern intelligent technology (IT). Traditionally, IT includes fuzzy logic $(\mathrm{FL})$, genetic algorithms $(\mathrm{GA})$ and neural networks $(\mathrm{NN})$.

As shown in recent studies, the IT could be successfully used during creation of complex system of control. Modern control device shall ensure proper facility control in different modes of its operation, be sustained both to sharp changes and slow degradation of control system parameters, to take into account possible presence of noises and external provided and unintended influences.

As the analysis of work in theory and in practice of artificial intelligent [1-30], for the current day the significant effective technologies of artificial intelligent have been created, which are applied in various practical applications, and in control as well. However, most authors use those technologies to design, research, and adopt local control systems, intended mainly to solve the problems of stabilization of some process variables.

The aim of this work is to develop methods and tools for the creation of intelligent hybrid systems of optimal process control.

\section{Development of methodology of creation of intelligent and hybrid systems of optimal process technologies control}

The department of automation and control of the K.I. Satpayev Kazakh National Technical University actively engaged in development research of hybrid and intelligent systems of various process technologies control [31-34]. Numerous studies conducted at the Department as well as analysis of publications [1-30] showed that IT could be used during directly development of model of optimal process control, not model of process technologies as itself. That means that the considered technologies enable to develop the control algorithms at once, in contrast to the traditional chain:

development of process model structure $\rightarrow$ carrying out experimental investigation at the facility $\rightarrow$ model identification $\rightarrow$ formulation of optimization task $\rightarrow$ selection of optimization method $\rightarrow$ development of optimal control algorithm. The traditional approach is long-term (sometimes several years), expensive, and not always successful way of creation of optimal control system.

IT application allows us to solve similar problems at once and as experience has shown quite successfully. The fact that artificial intelligence methods involve the using of knowledge, experience and intuition of human experts who are familiar with the subject. So, it uses the so-called effect of "ready knowledge." In contrast, the development of a mathematical model (the main component of the system) is the process of creating of "new knowledge", and therefore requires a sufficiently long time to carry out theoretical studies, as well as high material and labor costs for experimental studies and model identification.

In addition, experienced process-operators during long-time work learned to process under optimal conditions at different initial situations (and they often get success). The transfer of "ready knowledge" of human-experts to the knowledge base of intelligent system greatly simplifies the creation of intelligent systems and their operation eliminates the effect of the "human factor" in process control (these are properties of the human body such as: fatigue, not fast enough reaction, not enough psychological stability, drowsiness during monotonous work, little experience of young operators, and other causes).

Using the basic idea of the work (development of process control models instead of a process model) and by development of the existing IT methods, we propose the following three-stages of process on creation of the optimal control systems of technological processes (see Picture 1).

At the first stage it is produced a priori investigations of technological characteristics of controlled object on literary source, publications in periodicals, and factory technical documentation. As a rule, the existing processes had had to go through a long phase of research, experimental -industrial and industrial tests before they would be adopted into production. Surely there are still available materials of those researches, as well as attempts to create mathematical models of creation of mentioned process. It is necessary to carry out careful analysis of all this information in order to use it in the development of intelligent control systems. This is especially important during potential creation of hybrid control 
systems (HCS).

At this stage it is necessary to analyze the researched process as object of control with identification of input and output, controlled and uncontrolled, ruled and not ruled variables. Meanwhile, it is necessary to estimate object persistence through various channels, the object class (continuous or discrete), completeness degree of information on instance variables, operational range of instance variables and etc.

After careful analysis of the available information, it is necessary to make the structure of the future control system, which will significantly simplify further work.

At the second stage, it is developed a process control model. With assistance of experienced experts (processoperators, or engineering and technical personnel of workshop or factory) the main goal of control (equivalent of target function in optimization tasks) is being determined, which used to be familiar as rule, and which is usually trying to be reached by experienced operators. Then by the method of ranking from the general list of all types of the variables it is determined those, which are considered as main for the mentioned object (process) according to the opinion of the experts.

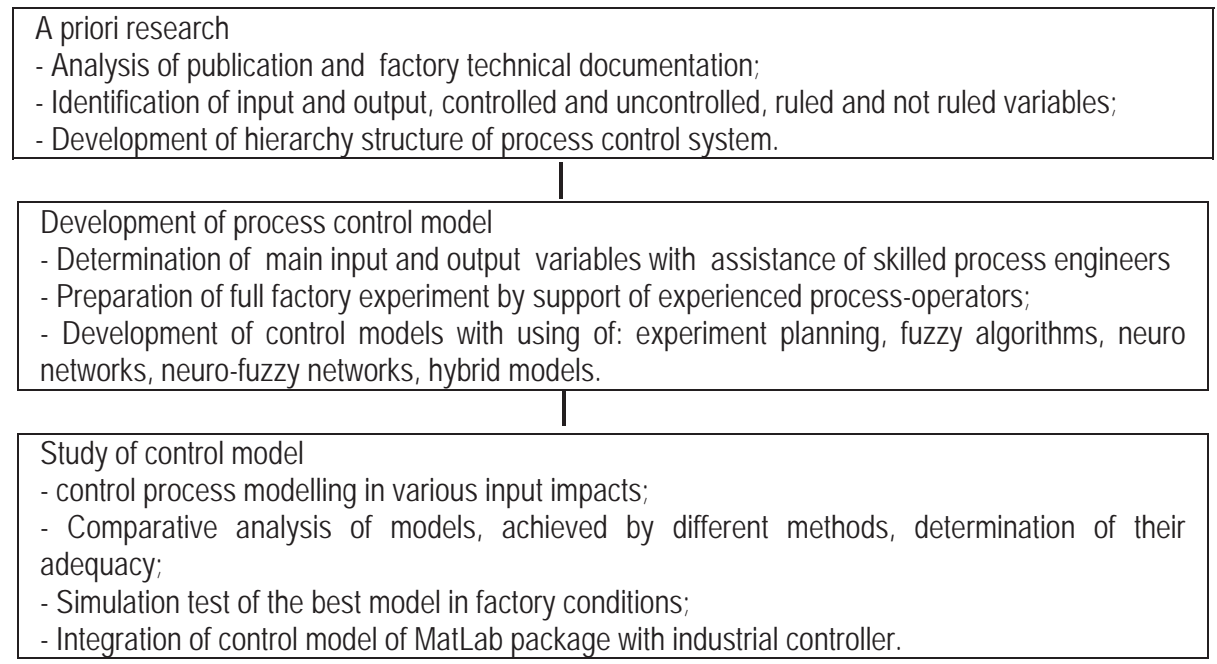

Picture 1: Three-step procedure of creation of hybrid and intelligent process technologies control system

The main task of the second stage is creation of matrix planning of full factorial experiment. By assistance of planning matrix of full factorial experiment it is made a model (algorithm) of object (process) control. Meanwhile, for example, for three-level factors the total number of possible combination of factors at two-input variables equals to $N=3^{2}=9$, for three variables $-3^{3}=27$ and etc.

For example, at two input variables it is made a planning matrix of full factorial experiment, specified in table 1. Tables of form 1 are considered as main for development of intelligent system due to long-term experience, knowledge, and intuition of experts in specified subject area. Quality of matrix of full factorial experiment will impact on efficiency of work of all control system.

Table 1: Planning matrix of full factorial experiment

\begin{tabular}{|c|c|c|c|}
\hline Experiment No. & $X_{1}$ & $X_{2}$ & $\begin{array}{c}Y^{\Im} \\
\text { Expert valuation }\end{array}$ \\
\hline 1 & 0,0 & 0,0 & \\
\hline 2 & 0,0 & 0,5 & \\
\hline 3 & 0,0 & 1,0 & \\
\hline 4 & 0.5 & 0,0 & \\
\hline 5 & 0.5 & 0,5 & \\
\hline 6 & 0.5 & 1,0 & \\
\hline 7 & 1,0 & 0,0 & \\
\hline 8 & 1,0 & 0,5 & \\
\hline 9 & 1,0 & 1,0 & \\
\hline
\end{tabular}

Values: 0,$0 ; 0,5 ; 1,0$ consider minimum, average and maximum values of the input variables $X_{1}$ and $X_{2}$. In the light of experience, knowledge and intuition of expert just need to put values of output variable $Y^{\ni}$ (command variable) in the 
range from 0,0 to 1,0 . Normalization in the range from 0 to 1 input and output variables is performed by the following formula:

$$
\bar{x}=\frac{x-x_{\min }}{x_{\max }-x_{\min }},
$$

Where: $\bar{x}$ - normalized (from 0 to 1 ) value of input or output variable; $\mathrm{x}$ - current value of variable; $\mathrm{x}_{\min }, \mathrm{x}_{\max }-$ minimum and maximum value of variable.

Preparation of matrix of planning of experiments is more convenient for experts, than making the fuzzy products rules, recommended in all guide books and publications. Meanwhile, expert does not need to invent endless terms: (very much, very-very little, quite normal and etc.) - expert just needs to put value of output (command) variable in the range from 0,0 to 1,0. Meanwhile a planning matrix of full factorial experiment could be used for four different methods of control model creation: planning of experiment, expert systems, neuro-networks, and neuro-fuzzy algorithms.

In contrast to the well-known classical method of experiment planning, the planning matrix of full factorial experiment with assistance of experts significantly speeds up and reduces the cost for this procedure. Experts carry out so-called "thought experiments" instead of expensive, conducted active experiments. Besides, it is necessary to take into account that conduction of active experiments in conditions of existing production is not possible due to potential emergency situations in case of changing of variables of process from minimum values to their maximum values and vice versa. Moreover there is no possibility to change the variables according to the planning matrix of full factorial experiment in many companies.

It is necessary to emphasize that the output values $\mathrm{Yi}$ are actually control variables, so planning matrix displays process control model for all combinations of input variables, scheduled by experts. In order to calculate intermediate values in combinations of the input variables (for example, $\mathrm{X} 1=\mathrm{X} 2=0.21$ and 0.74 ), it is needed to synthesize a process control model, which is considered as main task of the second stage.

It is necessary to mention that in conjunction with intelligent models the most effective is using well-known mathematical relationships, identified at the first stage of the research. At the same time it is necessary to make sure that such mathematical relationships adequately reflect those or other physical and chemical trends of a particular process. carried out.

At the third stage, it is conducted research of created control models. Meanwhile the following activities are

The obtained models are subject to careful research and analysis on their sensitivity, stability, and uniqueness. For this purpose a simulation of process control is performed in various changes of input variables, as well as curves of changes of output variables when the input variables change, and their analysis is carried out in conjunction with experts.

After completion of the research of models, obtained by different methods, the comparative analysis of their adequacy shall be performed as well. For this purpose it is calculated the output variables in the values of the input variables, taken from the planning matrix of full factorial experiment and compared with valuations, submitted by experts via the models. After that the comparative matrix is formed (see table 2), which could allow calculation of an extent of error of modelling by different methods. For example, absolute error in percentage is calculated by following formula:

$$
\delta=100 \frac{1}{N-1} \sum_{i=1}^{N}\left|Y^{j}-Y^{p}\right|
$$

where $Y^{3}$ and $Y^{p}$ - respectively experimental and calculated values of output variables.

Absolute error is calculated for the models, obtained in four different ways, after that their comparative analysis shall be carried out as well. The model with the lowest absolute error is considered to be the most adequate.

Table 2: Matrix of comparison of calculated and experimented output values

\begin{tabular}{|c|c|c|c|c|}
\hline Experiment No. & $X_{1}$ & $X_{2}$ & $\begin{array}{c}Y^{p} \\
\text { Model valuation }\end{array}$ & $\begin{array}{c}Y^{\ni} \\
\text { Expert valuation }\end{array}$ \\
\hline 1 & 0,0 & 0,0 & & \\
\hline 2 & 0,0 & 0,5 & & \\
\hline 3 & 0,0 & 1,0 & & \\
\hline 4 & 0.5 & 0,0 & & \\
\hline 5 & 0.5 & 0,5 & & \\
\hline 6 & 0.5 & 1,0 & & \\
\hline 7 & 1,0 & 0,0 & & \\
\hline 8 & 1,0 & 0,5 & & \\
\hline 9 & 1,0 & 1,0 & & \\
\hline
\end{tabular}


The most adequate model shall pass simulated tests in conditions of the current production. At the same time, input of the model shall be provided with valid input variables taken from metering devices of industrial unit, and the modelling results (output command variable) and compared with the values of control carried out by an experienced processoperator. In case of satisfactory result of simulation tests, integration of the model with an industrial controller shall be carried out. Otherwise, it starts all over again — a return to the first stage, and refinement of the model parameters as well. Let's consider the effectiveness of the proposed method by creating a system of optimal control of granulation (pelletizing) of bulk material as one of the most common processes in the metallurgical and chemical industries [35-47].

\section{The theoretical basis of pelletizing process}

For normal development of the granulation process of thin materials, their volume should have fluctuations in density and moisture content, so it requires the germinal centers. During pelletizing of fine materials, germinal centers are considered as some lumps whose pores are filled in with water. Lumps with sufficient weighing (several grams) during moldering in granulator shall obtain kinetic energy stores, which becomes enough for implementation of restructuring of pellets. Besides, the bulk material moistens up to maximum capillary water capacity condition and shall have a reduced strength of clutch, which makes easier reciprocal displacements of separate particles and obtain a dense structure of lump [37].

The process of formation of the nucleus and the formation of pellets with the "dry" granulation (when the material with humidity less than optimal and which is missing water amount as well is provided to pelletizer) could be represented as follows. A drop of water trapped in the layer of the material due to capillary forces at once begins to spread in all directions, filling the pores between the separate particles. Critical dimensions of the lump are directly proportional to the magnitude of the drop and inversely proportional to the porosity of the material layer. Water almost stops spreading inside the bulk material, as soon as the humidity reaches the maximum lump capillary capacity. This term takes a few seconds.

Obviously, the size of the lump is determined primarily by the size of water droplets falling into the layer. In the case of "wet" granulation (when the material with humidity more than optimal and also some amount of dry material are provided to pelletizer), germinal centers are made as a result of the destruction of "dried bread" (de-watered concentrate in vacuum filters).

Further lumps in pelletizer will get more compacted as result of multifold moldering and clashes to fixed material layer - separate particles due to reciprocal displacement get more compacted. Meanwhile, free water is squeezed out the surface of the lump, resulting in further connection with such lump of dry particles becomes possible. As the particles approach to each other the thickness of adhesive film becomes less, and adhesive strength increases. Due to the external dynamic effects an elastic resistance of water film is overcome, particles are captured by a molecular trap are and attracted to each other. It is required to stress that the approaching of particles by reducing of thickness of adsorbed films is possible only in case that the excess water is absorbed on the lump surface, for example as result of joining of new particles of material [37].

In this mode of operation of the granulator, there is a curtain minimum thickness of water films inside the lump corresponding to value of dynamic loads. As soon as this limit is reached, further water extraction on the lump surface will stop, pellet stops to grow and its strength will reach a maximum value.

Some authors [41] mention another reason for water extraction on the lump surface - under the centrifugal forces influence caused by lump rotation during its rolling down. Presented [37] approximate calculation proves that this phenomenon does not play any significant role.

For calculation of centrifugal force, an elementary capillary is released in a lump with length ${ }^{l_{0}}$, filled with water. At any moment of time, the pelletizing of lump could be represented as its rotation with curtain angular rate $\omega$ in respect to the point of contact the surface of pellet (point $K$ on picture 2). Meanwhile the water located at the diametrically opposite end of capillary suffers the biggest centrifugal force (at the point $B$ ). 


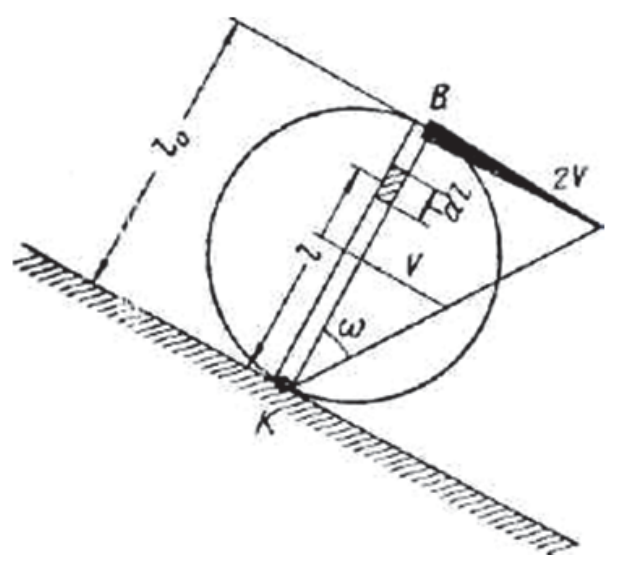

Picture 2: Scheme for calculation of value of centrifugal force during the lump rolling

The centrifugal force caused by rotation with the below mentioned value impacts on infinitesimal volume of water in the capillary $d l$

$$
d F_{\tilde{o}}=d m \omega^{2} l ; \quad d F_{\bar{o}}=\frac{\pi r^{2} \omega^{2} \gamma}{g} l d l .
$$

The total amount of centrifugal force, impacting on entire volume of water in the capillary equals

$$
F_{\ddot{o}}=\int_{0}^{l_{0}} \pi r^{2} \omega^{2} \frac{\gamma}{g} l d t ; \quad F_{o}=\frac{\omega^{2} \pi r^{2} \chi_{0}^{2}}{2 g} \text {. }
$$

Meanwhile pressure from centrifugal force impacts on the unit of cross-sectional area of the capillary

$$
P_{\ddot{o}}=\frac{\omega^{2} l_{0}^{2} \gamma}{2 g}=\left(\frac{V}{l_{0}}\right)^{2} \frac{l_{0}^{2} \gamma}{2 g} \text {. }
$$

During the pelletizing, the separate particles are getting closer at the distance of $10^{-4}-10^{-5} \mathrm{~mm}$, which causes the negative capillary pressure, which prevents the action for centrifugal force with value up to $15,0 \mathrm{n} / \mathrm{sm}^{2}$. It is natural that in the dry method of granulation the dimension of pellet could not be regulated by dimension of drops of water, falling into the layer of bulk material. In order to receive big pellets $(15-30 \mathrm{~mm})$, the quantity of contemporary incoming volume of water shall be equal to 1-4 $\mathrm{sm}^{3}$. Separate drops of such dimension could not be obtained. Meanwhile mealy and massive lumps obtained by such methods shall be ruined in the pelletizer under the influence of high dynamic loads or (at low efforts) will not obtain sufficient density and strength.

Therefore pellets with big sizes shall be obtained by sequential rolling of separate layers of material to the initial germ, caused during the falling of the drop with normal sizes to the material layer. For creation of water film of the pellets, providing the adhesion of dry small particles to the layer of germs it is required some quantity of water.

Therefore, in practice the predetermined quantity of water providing an optimal humidity is divided into two parts. The largest part shall be given to the layer of dry materials for creation of germs, and smallest part to the area of refilling of big sized pellets. In this case, pellets do not have homogeneous texture and they are not homogeneous, and consist of separate different layers.

In [37], it was given a theoretical and experimental analysis of behavior of bulk material in the rotating drum. It was found out that the pattern of movement of material in the rotating drum depends on its occupation degree, rotation speed and condition of inner surface of the drum. The authors [37] have determined three types of modes of material movement in cross-section of the drum: rolling mode, waterfall mode and cyclic mode (see Picture 3). 


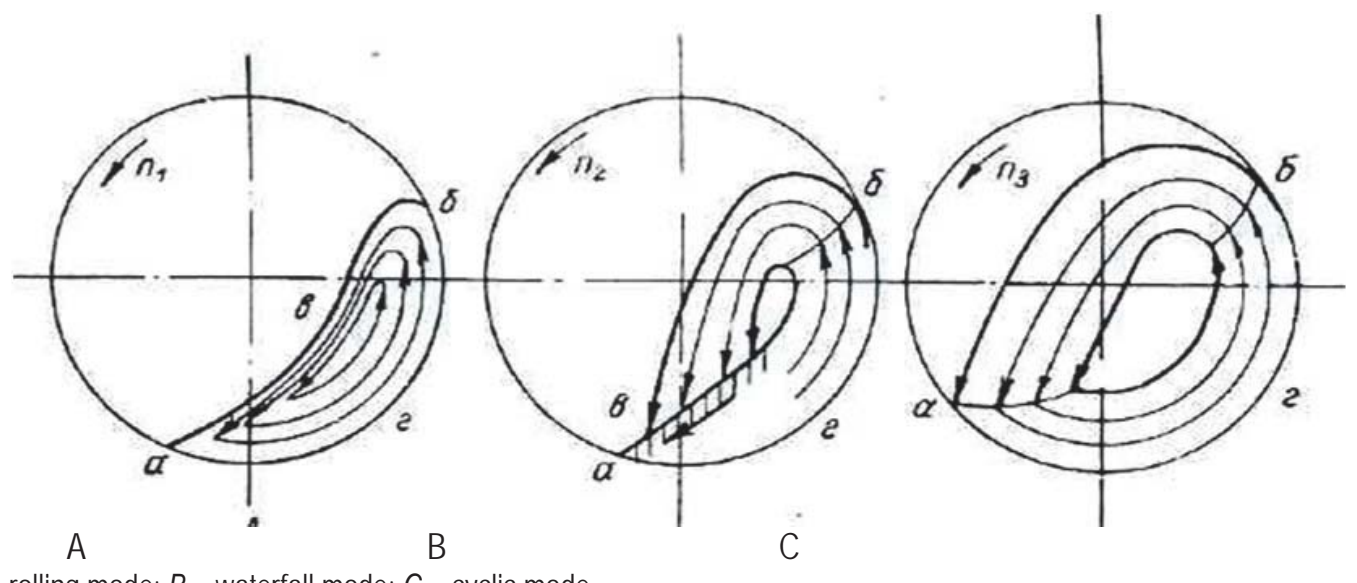

$A$ - rolling mode; $B$ - waterfall mode; $C$ - cyclic mode

Picture 3: Material movement mode.

During the rolling mode, as soon as the slope of surface of load exceeds the angle of repose, the excess material starts pouring down. In this mode, the loaded material is like rolling along the inner surface of the drum, at the same time spinning around the peculiar center - movement of material is performed in so-called rolling mode. The characteristic feature of material movement in the rolling mode is the absence of parabolic trajectory section on particles - after circular portion of route the material particles immediately transfers to the section of pouring (picture $3, A$ ). Volume of material during the movement in this mode is approximately bigger for $10 \%$ than the volume of loading in stall position.

Waterfall mode, observed at the high speeds of drum rotation (picture 3, B), has three sections of trajectory of the moving particles of bulk material: $a b$ - circular, $b c$ - parabolic and pouring section. In this case, the material after the circular section of the route leaves the surface of the drum and moves further through in the air along the parabolic trajectory. After falling, the material is poured down under the slope of the surface, formed by the remaining volume of material and then goes back to the circular section of trajectory. Therefore, the higher the speed of the drum rotation, the larger the obtained parabola. Volume, occupied by moving material is much larger of the same material in stall position.

Finally, at a sufficiently large number of rotations of the drum, the trajectories of separate elementary layers become closed and non-intersecting with each-other curves. In this mode of operation of the rotating drum the re-pouring section is lack - cycle of movement consists of circular $a b$ and parabolic $b c$ part of trajectory (picture $3, \mathrm{~B}$ ). Analysis of movement of material in cyclic mode shows that the gradient of speed of individual elementary streams is not big, separate layers are spinning with approximately the same angle speed.

It was found that the pelletizing of the sintering mix is most expedient to produce in the roll mode where there are absent the high impact loads, corresponding for waterfall and especially cyclic modes.

Particular interest is considered in the study of pattern of movement of bulk material along the axis of the rotating drum [37], since it effects on speed of movement of material along the axis $v_{i}$. According to this value, it is possible to calculate the residence time of material in the drum (i.e., quality of pellets) and its efficiency.

Upon reaching the top position, the material starts pouring down in layer of certain thickness on the slope, formed by rising material and inclined to the horizon on the plane of rotating under the angle $\omega$.

While operating the rotating drums in a continuous mode, this plane of pouring contemporary inclined to horizon and to the side of unloading end of the drum, as result during the pouring down each elementary volume of material contemporary moved at some distance and along the axis of the drum. This mechanism is specific for any cross-section of the drum. Therefore, the entire volume of bulk material, at the same time located in the drum could be divided into two parts, one of which is moving up on cyclic trajectories, and other pouring down. Meanwhile along the axis of the drum only a pouring part of material is moving on, the rest volume, rising upon the concentric circles is stationary in respect to ends of the drum. Such picture at continuously operation of the drum remains constant over time (pseudo stationary). This situation gives a rise to offer a different, more fundamentally correct circuit behavior of the material in the rotating drum. The movement of bulk material can be represented as a "slip" of layer of a certain thickness $h_{c}$, approximately equaling to the thickness of the pouring material along the inner surface of the drum under some angle $\gamma$ to a plane, which is perpendicular to the axis of rotating. In this case, the nature of movement of bulk material in the rotating drum is reduced to the regular movement of a body. Each elementary volume of material during the rotating of the drum 
describes on its inner surface a spiral (helix) with an elevation angle $\gamma$. This scheme allows us to derive a necessary calculation formula for characteristic of operation of rotating drums as process units.

If we intersect the drum by plane, which is perpendicular to the axis of rotation, than there will be a line of sliding layers with length $m$ and thickness ${ }^{h_{c}} M$ in section. This line will shift around circumference of drum at the distance $\pi D n M$ and along its axis - at the distance $\pi D n \operatorname{tg} \gamma_{M}$ in 1 min (picture 4), i.e., by crossover-

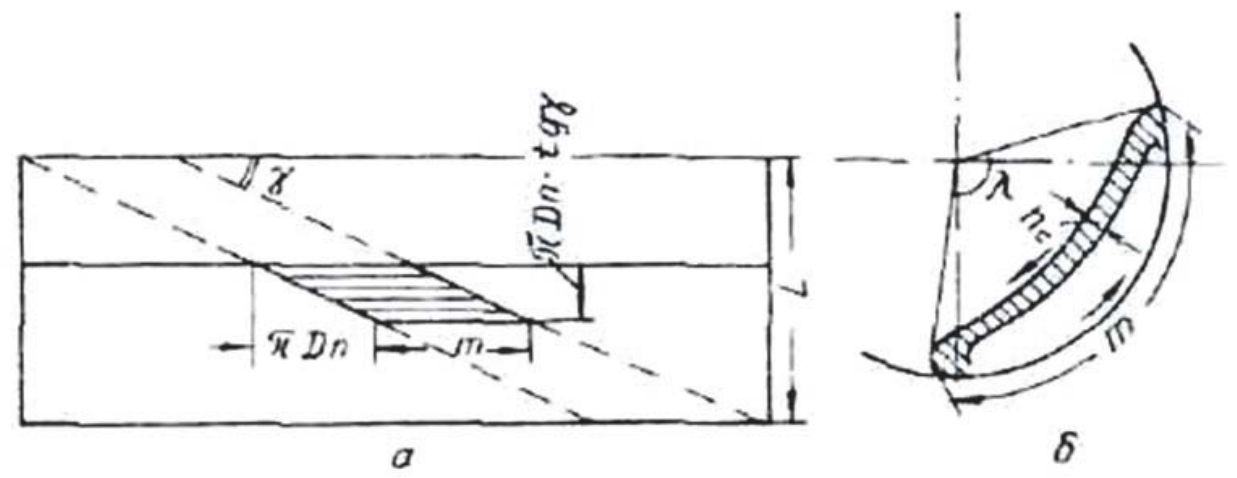

Picture 4: Scheme for development of formula of calculation of productivity of rotating drums: $a$ - development of cylindrical drum; $b$ - cross section of drum

section of drum per time unit it passes $Q M^{3}$ of material, that is voluminal productivity of drum:

$Q=\pi D n \operatorname{tg} z m h_{c} m^{3} / \min$

Because

$$
\begin{aligned}
& m=\frac{D}{2} \quad(\lambda-\text { in radians }) ; \\
& m=\lambda \frac{D}{2} \cdot \frac{2 \pi}{360} \quad(\lambda-\text { in degrees })
\end{aligned}
$$

and $h_{c}=(-0,011+0,00087 \lambda) D^{*}$, than after substitute and accounting of bulk mass ( $\left.\gamma_{\text {bulk }}\right)$ we have:

$G=\frac{\pi^{2} \gamma_{\text {iaĩ }}}{360} n D^{3} \lambda(0,00087 \lambda-0,011) \operatorname{tg} \gamma$.

The formula's analysis (9) shows that parameter that has strong influence on productivity of rotating drums, is diameter. If approximately similar mode of motion of material is kept, drum productivity becomes directly-proportional to cube of its diameter.

In order to use developed formula (9) practically it is required to know degree of filling of any section of the drum (by $\lambda$ ) and ${ }^{\operatorname{tg} \gamma}$. However these values, besides productivity also depends on $D, n$ and $\alpha$. Herewith in case of uninterruptible operation of rotating drum, such dependence is established automatically between specified parameters, where equation is right (9).

Main indicator, that determinates quality of pellets is duration of stay of part inside the drum, which is determined according to the formula [37]

$$
\tau_{i}=\frac{G_{i}}{G}=\frac{\Delta l_{i} \xi_{i} \pi D^{3} \gamma_{\text {bulk }}}{400 G}
$$

According to the formula (10), it is possible to calculate average time materials stay in the drum. However experimental researches [37] showed, that coarse particles moves along the drum axis with significant speed in comparison with small ones (30\% of difference). That explains the reason of aggregation of material on coarseness in cross section of the drum.

The most important characteristic of operating a rotating drum is the length of route, which is passed by material. This route presents as hypothesis of rectangular triangle for each surface element, i.e.,

$$
s_{i}=\frac{\Delta l_{i}}{\sin \gamma_{i}}
$$

It is shown in [37] that decrease in the inclination angle of a drum, if the same productivity is kept, is accompanied 
with increase of degree of its filling and relevant increase of duration of materials stay in the drum that shall improve the process of pelletization. On this point, horizontal drums with higher speed of rotation have the best indicators.

Therefore, studies have [37] determined productivity of pelletizer based on formula (9) in dependence on drum parameters (diameter, length, rotation speed) and quality of pelletization $(10,11)$, that depends on duration of stay of material in the drum and length of route, passed in the drum.

However, researchers [37] and formulas $(9,10,11)$ do not take into account material pelletization ability, experiments were carried out with use of polystyrene, as bulk material. Polystyrene allows the author [37] to check accuracy of formulas $(9,10$ and 11) on productivity, duration of stay and passed route, however he could not describe the pelletization process itself with the help of these dependences.

Materials' pelletization abilities can be evaluated by granulation coefficient [37]; numerically it can be evaluated by the following method. It is known, that because of poor quantity of coarse grains (nucleation) in initial charge material, pelletization process is unstable, accordingly granulation process-rolling of fines over coarse grains is destructed, there is simple mixing of material in pelletizator. In accordance with the abovementioned, granulation pelletization ability of material can be evaluated by coefficient, which represents relation of surface of palletization fractions to the volume of fine (palletized) fractions

$$
\frac{\frac{6}{\gamma} \sum_{i=1}^{i=n} \frac{P_{i}}{d_{i}}}{\frac{P_{i}}{\gamma_{i a ̃ n}}}
$$

The more this relation is, the faster fine will be rolld in coarse grains. Due to this, the suggested indicator reasonably can be called granulation speed coefficient $C_{g s}$. Because for the most part of magnetitic materials $\gamma$ is around Ybulk, than

$$
\mathrm{C}_{\mathrm{gs}}=6 \frac{\frac{P_{1}(\%)}{d_{1}}+\frac{P_{2}(\%)}{d_{2}}+\ldots+\frac{P_{n}(\%)}{d_{n}}}{P_{i}}
$$

Use of this indicator gives the possibility to explain unusual, at first sight, results of tests on pelletization, where Cherepovets charge materials with $20 \%$ of the return with coarseness of 1-3 $\mathrm{mm}$ after pelletization had higher gas permeability than the same charge material with the same amount of return, but with coarseness of 5-12 $\mathrm{mm}$. When coarse return was replaced with fine one, number of pelletization centers was significantly increased, surface area of palletized fraction was also significantly increased. When $20 \%$ return with a coarseness of 5-12 $\mathrm{mm}$ was replaced with the same amount of return with coarseness of $1-3 \mathrm{~mm}, C_{g s}$ is increased in 40 times (from 0,18 up to 0,75) in considered tests. That is exactly how positive influence of breakage of return on charge material gas permeability shall be explained $[40,42]$.

\section{Development of Structure and Control Systems}

On the basis of peculiarities of the pelletization process as an object of control, we would like to suggest structure of system of control over this process, which provides optimal keeping of modes. The main complication of control over pelletization process is in hard conditions on quality of final pelletized material if there is non-stationary quality and quantity composition of charged components. As input variables, accounted during calculation of pelletization modes, we consider, as follows:

- granulometric composition of phosphate fines, cox, flux, dust, secondary, and primary return;

- percentage composition of charge components .

- Control variables can be:

- proportion of charge materials-water;

- -drum rotation speed;

- load (productivity) of drum.

It is also required to hold the most optimal rerolling mode of motion of material in drum in order to provide quality of final product. Taking into account input and output variables, we would like to suggest the following structure of control (see the picture 5). Main peculiarity of suggested structure is possibility to change tasks by regulator periodically - due to production of regular Working pair of bunker, and transfer into new pair with other, already known quality characteristics 
of charge material.

The optimal control system represents double-level hierarchic structure. Calculation of optimal modes of process carrying out for regular pair of bunkers, which are ready for operation at upper level. There are regulators of the current automation system, for which optimal tasks are calculated, at the lower level. It means principal of combined control is realized- disturbance control, and lower level realizes deviation control. As previously mentioned, quality of pellet strongly depends on granulometric composition of components of charge, load and drum rotation speed, amount of supplied water.

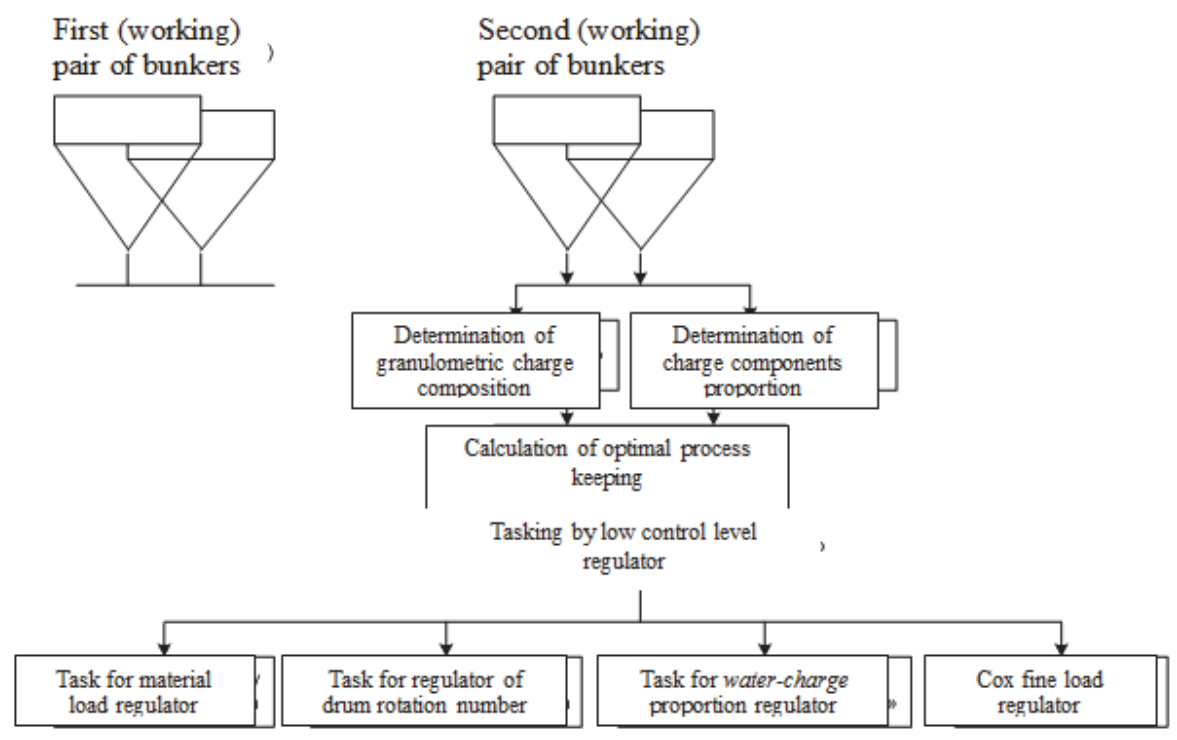

Picture 5: Structure of optimal control system

All these dependences with one or another level of accuracy are described mathematically in different researches [3646]. However, the most important dependence: pelletizing ability of charge particles $\rightarrow$ coarseness and hardness of pellets is not described till now. It is connected with extremely complicated physico-chemical processes, which are carried out at the border of solid and liquid phases.

\section{Mathematical description of influence of different process factors on quality of pelletization}

Interdependence between productivity, drum rotation speed, and bulk weight of material is fully described by the formula (9), where $n$ - number of drum rotations; $D$-drum diameter; $\quad \mathrm{Y}$ - angle of material rise in rotating drum according to cylinder element of drum (see the picture 4); үbulk - bulk weight of material; $\lambda$ - angle, which is dependent on angle of bulk $\omega$, herewith

$$
\lambda=180-2 \omega \text {. }
$$

It is necessary to note that during uninterruptible drum operation there is automatic establishment of such dependence, where equation (9) is right.

Equation (14) determines maximum degree of drum filling, up to which, material will move in rerolling mode. First of all angle $\omega$ is determined by angle of material internal friction (angle of nature friction) in equation (15), it strongly depends on drum rotation speed. In case of other equal conditions angle of bulk $\omega$ extends with increase of degree of drum filling.

Taking into account dynamic load, angle $\omega$ come out at 10-25 degrees higher than angle of nature friction. The higher drum rotation speed is, the more angle $\omega$ becomes, the less maximum filling degree is. However equation (14) can be used only for not exceeding $10 \%$ degree of filling.

The critical value of drum rotation speed, which characterizes border between rerolling mode and waterfall mode, is determined based on formula [37] if filling degree is high: 
$n_{k p}=30 \sqrt{\frac{-\cos \left(10+\frac{\lambda}{2}\right)}{R}}$,

where $R$ - drum radius.

One of the main parameters is material speed of movement along the drum axis $V_{L}$, according to which we can calculate drum productivity

$G=v_{L} F \gamma$

where $F$ - cross section area of material in drum; $\gamma$ - density of material.

The most complicated thing is determination of speed $v_{L}$. There are many formulas for its calculation in literature; however, their accuracy is quite low. In author's opinion, the most accurate determination of drum productivity shall be better defined based on formula (14).

As it was already mentioned, effectives of granulation is pretty much characterized by granulation speed coefficient [37]:

$$
C_{g s}=\frac{6\left[\frac{P_{1}(\%)}{d_{1} \gamma_{1}}+\frac{P_{2}(\%)}{d_{2} \gamma_{2}}+\ldots+\frac{P_{n}(\%)}{d_{n} \gamma_{n}}\right]}{\frac{P_{n}}{\gamma_{\text {bulk }}}}
$$

where $\gamma_{i}$-specific density of charge components;

$P_{i}$ - content $i$ component in charge (pelletizing fractions);

$P_{n}$-concentration of fine (pelletized) fractions;

$\gamma_{\text {bulk }}$ - bulk weight of pelletized fractions.

Formula (17) represents proportion of surface of pelletizing fractions to the volume of fine (pelletized) fractions.

However there are many variables which are determined complicatedly in formulas $(9,14-17)$, as follows: $\gamma_{i}, \gamma_{\text {bulk, }}$ $\omega, \gamma$, which can be hardly measured operatively, and for determination of angles $\omega$ and $\gamma$ it is required to carry out quite complicated and expensive experiments.

Besides that, equation (17) determines the coefficient of granulation effectiveness, which has no relation to equation (9,14-17). It means that equation (17) determines the quality of granulation only as indicator of its effectiveness; its numerical value does not participate in calculation of outlet variables.

However equations $(9,14,15)$ reveal main dependences between inlet and outlet variables. It gives the possibility to get over mentioned indeterminacies with the help of contemporary mathematic tool-artificial intelligence techniques. In contrast from traditional mathematic modelling of physic-chemical processes, carried out in drum pelletizer, new tools allow modelling of the control process itself. In other words, passing stages of construction of model structure, its identification and development of optimal control algorithm, intellectual technologies allows to start development of control process model itself at once and on the basis of many years' experience of process operators.

\section{Synthesis and research of intellectual models of pelletizing control process algorithms}

During development of intellectual model of pelletization control process algorithms, we have accepted the following allowances:

- due to the fact that during primary mixing there is a quite good intermixture of charge components, process parameters: $\gamma_{b u l k}, \gamma_{i}$ and $\omega$ have constant values, and they are determined by experimental method;

- effectiveness of pelletization basically depends on the value of granulation coefficient (formula 13);

- effectiveness of pelletization depends not only on granulometric composition of charge material, but also on percentage composition of charge components (phosphate fines, return, cox fines, dust and etc.). In connection with the fact that charge material has more than $90 \%$ of phosphate fine and return, we will take into account only these two components of charge;

- optimal charge-water proportion first of all depends on proportion of coarse and small fractions - the smaller fractions are, the more humidity they need for agglutination of charge particles. The best evaluation of such proportion can be granulation speed coefficient, which is determined based on formula (18). The less this coefficient value is, the more humidity it needs in order to provide good agglutination of charge pellets (i.e., 
charge-water proportion will have less value);

- besides that, the amount of humidity required for provision of qualitative agglutination of charge particles, also depends on percentage composition of charge components (phosphate fine and return);

- consider humidity of initial material as constant.

Inlet and outlet (control) variables were determined above. Herewith the main purpose of intellectual model is determination of such productivity of pelletizer, that would provide high quality of produced pellets and optimal chargewater proportion for provision of charge pellets with agglutination. As it is seen from such setting of control task, the main purpose of this model is producing high-quality final products, on which quality of agglomerate is dependent.

The following stage of construction of intellectual model is the formation of rules base (knowledge base) by questioning of experienced process operators, who has been working with drum pelletizer for a long time. Taking into account accepted allowances, conducted questioning allows to form rules as matrix of planning of Complete Factorial Experiment with an interval step that equals to 0,25 , specified in the table 3 , for outlet (control) variable - drum productivity.

Table 3: Matrix of Complete Factorial Experiment planning

\begin{tabular}{|c|c|c|c|}
\hline $\mathrm{N}$ & Granulation coefficient, C & Amount of return, B & Drum productivity, G \\
\hline 1 & 0,00 & 0,00 & 0,03 \\
2 & 0,25 & 0,00 & 0,15 \\
3 & 0.50 & 0,00 & 0,25 \\
4 & 0.75 & 0,00 & 0,27 \\
5 & 1,0 & 0,00 & 0,3 \\
\hline 6 & 0,00 & 0,25 & 0,06 \\
7 & 0,25 & 0,25 & 0,42 \\
8 & 0.50 & 0,25 & 0,62 \\
9 & 0.7 & 0.25 & 0,7 \\
10 & 1,0 & 0.25 & 0,73 \\
\hline 11 & 0,00 & 0,50 & 0,12 \\
12 & 0,25 & 0,50 & 0,56 \\
13 & 0,50 & 0,50 & 0,87 \\
14 & 0.75 & 0.50 & 0,9 \\
15 & 1,0 & 0,50 & 0,95 \\
\hline 16 & 0,00 & 0,75 & 0,09 \\
17 & 0,25 & 0,75 & 0,5 \\
18 & 0.50 & 0,75 & 0,75 \\
19 & 0.75 & 0,75 & 0,85 \\
20 & 1,0 & 1,00 & 0,88 \\
\hline 21 & 0,00 & 1,00 & 0,07 \\
22 & 0,25 & 1,00 & 0,43 \\
23 & 0.50 & 1,00 & 0,62 \\
24 & 0.75 & 1,00 & 0,72 \\
25 & 1,0 & & 0,88 \\
\hline
\end{tabular}

The Matrix of Complete Factorial Experiment planning is essential for the synthesis of intellectual control models. Three types of intellectual models were synthesized by us, as follows: fuzzy, neural network, and neuro fuzzy. Synthesis of these models is carried out with the help of familiar package Matlab.

Neural network and fuzzy models, as well as neuro fuzzy networks satisfy terms of integration into this control system, but in order to find out what are the best, it is better to carry out their comparison. Results that were obtained above are specified in the table 4. 
Table 4: Comparison analysis of model adequacy

\begin{tabular}{|c|c|c|c|c|}
\hline $\mathrm{N}$ & Fuzzy logic & Neuro network & Neuro fuzzy network & The correct answer \\
\hline 1 & 0,08 & 0,05 & 0,04 & 0,03 \\
2 & 0,15 & 0,15 & 0,15 & 0,15 \\
3 & 0,25 & 0,25 & 0,25 & 0,25 \\
4 & 0,25 & 0,27 & 0,27 & 0,27 \\
5 & 0,4 & 0,3 & 0,3 & 0,3 \\
\hline 6 & 0,08 & 0,07 & 0,05 & 0,06 \\
7 & 0,4 & 0,41 & 0,41 & 0,42 \\
8 & 0,5 & 0,55 & 0,59 & 0,62 \\
9 & 0,75 & 0,75 & 0,73 & 0,7 \\
10 & 0,75 & 0,75 & 0,75 & 0,73 \\
\hline 11 & 0,25 & 0,12 & 0,12 & 0,12 \\
12 & 0,5 & 0,5 & 0,55 & 0,56 \\
13 & 0,75 & 0,85 & 0,85 & 0,87 \\
14 & 0,92 & 0,92 & 0,92 & 0,9 \\
15 & 0,93 & 0,93 & 0,93 & 0,95 \\
\hline 16 & 0,08 & 0,08 & 0,09 & 0,09 \\
17 & 0,5 & 0,5 & 0,5 & 0,5 \\
18 & 0,75 & 0,75 & 0,75 & 0,75 \\
19 & 0,75 & 0,80 & 0,83 & 0,85 \\
20 & 0,92 & 0,88 & 0,88 & 0,88 \\
\hline 21 & 0,08 & 0,08 & 0,08 & 0,07 \\
22 & 0,5 & 0,5 & 0,45 & 0,43 \\
23 & 0,6 & 0,6 & 0,6 & 0,62 \\
24 & 0,75 & 0,72 & 0,72 & 0,72 \\
25 & 0,92 & 0,92 & 0,90 & 0,88 \\
\hline Absolut error & $4,5 \%$ & $2,08 \%$ & $1,125 \%$ & \\
\hline
\end{tabular}

As it is seen from Table 4 , the best result was shown by neuro fuzzy model- error $=1,125 \%$.

The curve line-1, obtained during modelling of neuro fuzzy model with step of 0,25 is shown at the picture 6 . During comparison of results of calculations in points: 0,$0 ; 0,25 ; 0,5 ; 0,75$ and 1,0 if $B=0,5$ (see the table 3 for $\mathrm{N}=11$, $12, \ldots, 15)$ quite high convergence of results, close to $1,1 \%$ was obtained.

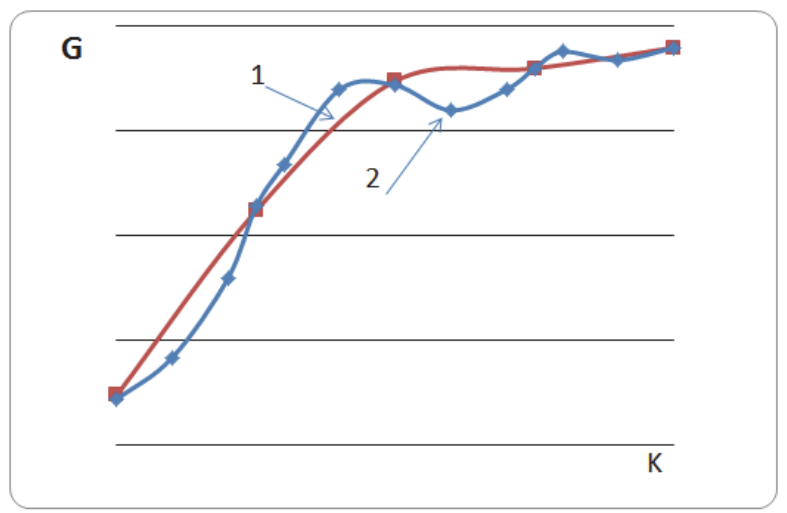

Picture 6: Results of modelling of neuro fuzzy model, trained on 25 experimental points. Curve line-1 with modelling step $=0,25$. Curve line-2 with modelling step $=0,1$.

However, during modelling of the same model by 0,1 interval, we obtained curve line -2 , which has significant difference from curve line -1 in intermediate points. Herewith comparison of the curve line-1 and the curve line-2 in points with step 0,1 makes error in $2,67 \%$.

In order to reduce modelling errors, matrix of Complete Factorial Experiment planning for 65 points was developed and retraining of neuro fuzzy network was conducted already on 65 points for different B values. As the result, absolute 
error value was reduced till $0,8 \%$. It is shown at the picture 7 that curvline-1 and curveline-2 are practically coincided for $B=0,5$.

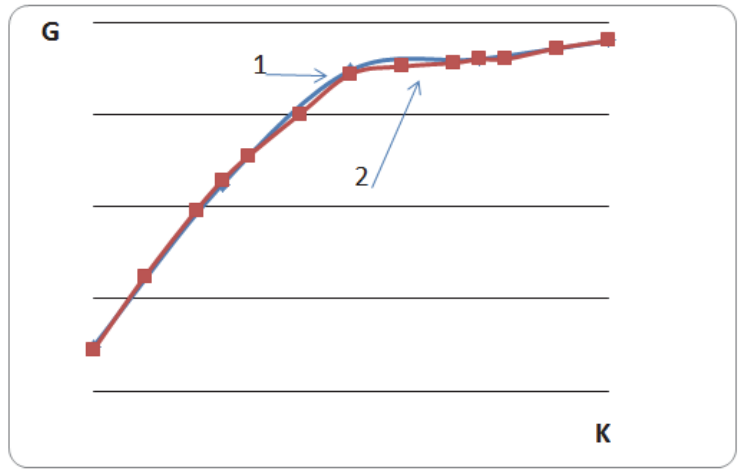

Picture 7: Results of modelling of neuro fuzzy model, trained on 65 points

Analogic picture is displayed and when quantity of $B$ return is changed with step equals to: 0,$0 ; 0,25 ; 0.5 ; 0,75$ and 1,0 if $\mathrm{C}=0,5$, represented by the curveline- 1 at the picture 8 . Modelling with interval 0,1 gives curve line-2, which is very significantly differ from the curve line-1. Both curve lines after retraining of the neuro-fuzzy network on 65 points are shown at the picture 9 .

G

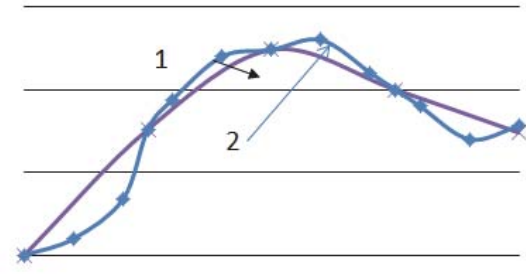

Picture 8: Results of modelling of granulator productivity if amount of return on model, trained on 25 points is changed

G

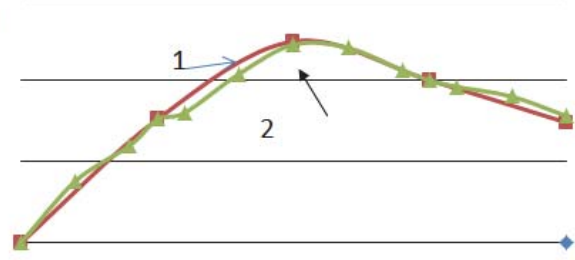

Picture 9: Results of modelling of granulator productivity if amount of return after retraining of network on 65 points is changed

Therefore, we may propose the following procedure of training and retraining of intellectual models. At first, the network is trained on the matrix of Complete Factorial Experiment planning with enough rough step of change of inlet and outlet variables, for example by 0,25 . Then it is required to carry out modelling for different combinations of inlet variables with smaller step (for example, after 0,1), according to which results we evaluate adequateness, and if it is necessary, network is retrained on bigger amount of points. Herewith values for retraining of network are produced by the method of the 
curve line-1 approximation for intermediate points (with step of 0,1). Such double-stage procedure allows retraining of network without experts involving, that significantly easy up their work.

After finding out by neuro fuzzy model of optimal granulator productivity $(G)$, such number of drum rotations is determined (n) according to proportion (14), which provides more favorable mode of materials movement in the drum rerolling.

\section{Conclusion}

The following conclusions can be made as a result of executed studies and obtained results.

A new method of development of intelligent and hybrid models (algorithms) of optimal management of processes, which includes a three-stage procedure of their development, was proposed. This method has been tested in the development of optimal management of process of pelletization of phosphate fines. All three stages (see picture 1) of proposed method were used during this procedure.

According to the first stage - A priori studies, the following results were found:

- that at the moment there are quite effective equations to describe the process of transferring of material in the granulator, depending on the speed of its rotation and the angle of inclination of the drum to the horizon;

- that rerolling regime, that allows to obtain the better quality of pellets is the most optimal;

- that the relationship between performance, rotation speed of the drum and bulk density of the material are most fully described by using of equation (14);

- that the granulation efficiency is largely characterized by coefficient of granulation speed (16);

- that the formulas (14 - 16) contain a lot of variables that are difficult to define, and which are very difficult to measure operatively, and it is necessary to carry out quite complicated and expensive experiments to determine the angles $\omega$ and $\gamma$;

- that equations (14 - 16) identify the main dependencies between inlet and outlet variables that give an opportunity to overcome the uncertainty by using of modern mathematical device-artificial intelligence methods.

The following work was carried out under the second stage - Development of control process model:

- matrix of planning of complete factorial experiment for development of control models over granulation process were developed with the help of experienced process operators of Novozhambul phosphorus plant, in this case as inlet variables were used: coefficient of granulation and the amount of return; and as the outlet (control) variables: drum productivity and the proportion of charge/humidity;

- fuzzy, neural, and neuro fuzzy models (algorithms) of pelletization process control were developed.

The comparative analysis of the developed models was carried out under the third stage - Research of control models:

- best results were obtained with the use of neuro fuzzy algorithms;

- carrying out simulations of three intellectual models with obtaining of corresponding curves do not contradict the physical and chemical regulations of granulation process;

- retraining of models with taking into account of intermediate points significantly reduces the values of the absolute errors, even for quite complicated (extreme) curves (see the picture 11 and 12);

- use of extra polished data for intermediate points for retraining allows great improvement of the quality of intellectual models without the help of experts.

Students with Master's and doctoral degrees of the Automation and Control Chair of the K.I. Satpayev Kazakh National University were actively involved in work on development of algorithm of optimal control over pelletization process of phosphate fines, for what the author expresses them his gratitude.

\section{References}

V.D. Gaskarov. Dialog procedures in preventive control and management systems Informational work by vehicles.: Collection book of scientific works Saint-Petersburg: Saint-Petersburg State University of water communications 1996. - 248-258 pages.

A.V. Leonenkov Fuzzy modelling in MAELAB and fuzzTECH environment. Saint-Petersburg.: BHV-Petersburg, 2003.736 p.:

T.A. Gavrilova, V.F.Khoroshevskiy Knowledge bases of intellectual systems. Saint-Petersburg, 2000. 348 p.

L. Rutkovskiy, Methods and technologies of artificial intellect. M.: Hot line-Telecom. 2010, 202-279p.

Tadeusiewicz R., Sieci Neuronowe, Akademicka Oficyna Wydawnicza RM, Warszawa 1993.

Zurada J., Introduction to Artificial Neural Systems, West Publishing Company, 1994. 
Haykin S., Neural Networks: A Comprehensive Foundation, Macmillan College Publishing Company, New York 1994

Hop Ch.M., Neural Networks for Pattern Recognition, Oxford University Press, Oxford, New York 1995.

Kacprzyk J., Zbiory Rozmyte w Analizie Systemowej, Wydawnictwa Naukowe PWN, Waarszawa 1986.

Zadeh L.A., Fuzzy sets, Information and Control, vol. 8, 338-353, 1965.

Fodor J.C., On fuzzy implication, Fuzzy sets and systems, vol. 42, 293-300, 1991.

Takagi T., Sugeno M., Fuzzy identificationof systems and its application to modeling and control, IEEE Trans. Syst., Man, Cybern., vol. 15, 116-132, 1985.

Kay S.M., Modern Spectral Estimation. Theory and Application,Prentice-Hall, Englewood Cliffs, New Jersey 1988.

Marple S.L.Jr., Digital Spectral Analysis with applications, Prentice-Hall, Englewood Cliffs, New Jersey 1987.

Rutkowski L., Filtry Adaptacyjne iAdaptacyjne Przetwarzanie Sygnalow: Teoria I Zatosowania, Wydawnicto Naukowo-Techniczne WNT, Warszawa 1994.

Zadeh, L.A. "Is there a need for fuzzy logic?" NAFIPS 20082008 Annual Meeting of the North American Fuzzy Information Processing Society-2008

D. Ramot, M. Friedman, G. Langholz, A. Kandel, "Complex fuzzy logic" IEEE Transactions on Fuzzy Systems, No. 11-2003

F. Bobillo, U. Straccia. FuzzyDL: An expressive fuzzy description logic reasoned. 2008 IEEE International Conference on Fuzzy Systems (IEEE World Congress on Computational Intelligence) 2008.

S. Russell, P. Norvig. Artificial Intelligence: A Modern Approach, 3rd edition. Prentice Hall. 2009

A. Mayrhauser, R. France, M. Scheetz, E. Dahlman, Generating test-cases from an object-oriented model with an artificial-intelligence planning system. IEEE Transactions on Reliability.No.49-2000

R.J. Solomonoff, Some recent work in artificial intelligence. Proceedings of the IEEE.No.56-1966

J. Gonzalez, S. DesJardins. Artificial Neural Networks: A New Approach to Predicting Application Behavior. Research in Higher Education.No.43-2002., 235-258

C. Lim, L. Jain. Advances in Swarm Intelligence. INNOVATIONS IN SWARM INTELLIGENCE. No. 248., 2009. 1-7.

C. Ramos, J. Augusto, D. Shapiro. Ambient Intelligence-the Next Step for Artificial Intelligence. IEEE Intelligent Systems. No.23. 2008. 15-18.

S. Kalogirou. Artificial intelligence for the modeling and control of combustion processes: a review. 2003. 515-566.

I.M. Makarov, V.M. Lokhin, S.V. Manko and others. Artificial intellect and intellectual control systems. M.:science.-2006.

V.I.Vasilyev, B.G. Inlyasova Intellectual control systems. Theory and practise. M.: Radiotechnics.-2009.

Li Y., Ang K.H., Chong G.C.Y. Patens, software and hardware for PID control: an overview and analysis of the current art IEEE Control System Magazine. -2006.-V.26-No.1.-P.42-54.

Ponce-Cruz P., Ramirez Figueroa F.D. Intelligent control systems with Lab VIEW. Berlin:Springer Verglad.-2010.

B.A. Suleymenov, G.M. Mutanov. Control over process in non-ferrous metallurgy. Almaty: Gylym. 1997. 279 pages

B.A. Suleymenov. Intellectual and hybrid systems of control over processes. Almaty, 2009. 207-304 pages.

B.A. Suleymenov, T.T. Shuvatov. Method of construction of GCU diagnostic systems. Engineering and technical magazine Automation Reporter, No.30, 2010, 12-16 pages.

A.B. Suleymenov, M.Sh. Kadenov, B.Sh. Kadenov. Development of hybrid system of control over pelletizing of agglomerated charge. Engineering and technical magazine Automation Reporter, No.31, 2011, 5-9 pages.

A.B. Suleymenov, D.Zh. Hammedov Development of MES-technology-for agglomeration division of the Novo Dzhambul Phosphorous Plant. Engineering and technical magazine Automation Reporter, No. 3(33), 2011 г., 10-13 pages.

N.S. Shumakov, A.M. Kunayev. Agglomeration of phosphorites, Almaty: Science, 1982, 264 page.

N.S. Shumakov, G.A.Pekhotin. Calculation of material and thermal balance of phosphorites agglomeration process. Department of the scientific and research division of TEchem, No.1380/77.

V.I..Korotich Theoretical bases of pelletization of iron-oxide, M.: Metallurgy, 1965. 150 page.

I.I.Rovenskiy, Work of Scientific and Technical Society of the ferrous metallurgy, t. 22. Metallurgy, 1959.

N.N.Berezhnoy, G.V.Gubin, L.A.Drozhilov, Pelletizing of fine-grained pellet plant feeds , M., 1971, 174 page

D.G.Khokhlov, A.P.Yakobin, Production of fluxed agglomerate, Sverdlovsk. Metallurgy, 1959.

Tigerschiold M., Ilmoni P. A., Amer Inst. Min.Eng. Proceedings of the Dlast Furnace and Coke Oven, Raw Materials Conference, v9, 1950, 18 p.

D.G.Khokhlov, A.P.Yakobin, Production of fluxed agglomerate, Sverdlovsk. Metallurgy, 1959.

A.Ph. Lebedev, Ground compaction if humidity is different. Stroyvoenmorizdat, 1949.

V.I..Korotich News of Higher Educational Institutions, ferrous metallurgy, 1963, No.11.

V.V.Tovarov, 1947, No.3.

B.V.Deryagin and others. Determination of specific surface of powdered solids on filtration resistance of expanded air. Published by Academy of Sciences of the USSR, 1957.

N.N.Berezhnoy, G.V.Gubin, L.A.Drozhilov, Pelletizing of fine-grained pellet plant feeds, M., 1971, 174 page 